\title{
Effects of Hot Rolling and Subsequent Tempering on Particle Erosion Behavior of SUS403 Martensitic Stainless Steel
}

\author{
Dong-Cherng Wen* \\ Department of Mechanical Engineering, China Institute of Technology, Nankang, Taipei, Taiwan, R. O. China
}

\begin{abstract}
The effects of hot rolling and subsequent tempering on particle erosion behavior of SUS403 MSS were investigated as a function of impact angle, reduction and grain size. The results indicated that, cutting is the dominant wear mode for material removal at oblique impact angle. At the higher impact angle, the mode is based on extrusion and cracking. However, at the medium impact angle, the wear is dominated by mode mixed with cutting and extrusion. The characteristics of the cracking are more obvious with increasing impact angle. The erosion rates of all reduction samples increase first and then decrease with increasing impact angle. The maximum erosion rate occurs at the angle of $45^{\circ}$. After hot rolling, grain refining has an effect on decreasing erosion rates in comparison to the direct quenched and tempered samples. Fine grain associated with high density of grain boundary and with strengthening effect in mechanical properties retards the cracking and then decreases the erosion rate. The effect of grain refining on decreasing erosion rate is more obvious with increasing both the impact angle and the reduction. At the normal impact angle, the percentage of decrement of erosion rate is approximately $45 \%$ when the material was rolled with $50 \%$ reduction. Increasing in the mechanical properties including hardness, ductility, tensile strength and toughness are contributed to decreasing the erosion rate. [doi: $10.2320 /$ matertrans.47.1975]
\end{abstract}

(Received April 10, 2006; Accepted June 7, 2006; Published August 15, 2006)

Keywords: hot rolling, tempering, particle erosion, stainless steel, grain refining

\section{Introduction}

Martensitic stainless steels (MSS) are widely used in application for which a combination of moderate corrosion resistance and high strength is needed, for example, such as pump bodies, pipe fittings and turbine blades. Due to impact of hard and abrasive particles entrained in a fluid stream at high velocity, erosion is a common problem in these parts. Reducing the erosion wear of materials requires an understanding of the wear mode by which the material is removed. Two models have been employed to describe the erosion behavior for ductile materials and brittle materials respectively. Ductile erosion occurs by ploughing, micromachining and cutting, ${ }^{1-4)}$ and maximum erosion rates occur for impact at $\sim 20-30^{\circ}$. Brittle erosion occurs by initiation and propagation of cracks induced by erodent particle indentation, 5,6) and maximum erosion rates occur for impact at $90^{\circ}$. The erosion rate of material neither very soft nor very hard is generally exhibited as the mixture of these two models. ${ }^{7)}$ Brittle responses predominated at high impact angle, whereas ductile responses predominated at low impact angle. The angle of maximum erosion rates increases with increasing hardness of the materials. ${ }^{8,9)}$

The influence of mechanical properties on erosion rates of materials have been studied extensively. ${ }^{10-12)}$ In general, erosion rates of material are not consistently correlated with any single mechanical properties. Hardness, ductility, yield strength and other properties are though to affect erosion damage sometimes with the mixed effects of these properties. ${ }^{13)}$ Mechanical or thermal treatments can alter the mechanical properties of the material. The effects of these processes on erosion rate have been discussed in detail. Naim and Bahadur ${ }^{14)}$ reported that a higher erosion rate was observed for both the oblique and the normal impact conditions due to an increase in cold working. Goretta ${ }^{15)}$

*Corresponding author, E-mail: dcwen@cc.chit.edu.tw indicated that work hardening improved the erosion resistance of the copper. Bregliozzi ${ }^{16)}$ investigated the cavitation wear of austenitic stainless steels with different grain size. He concluded that the resistance to cavitation erosion increases with decreasing grain size. Korshunov ${ }^{17)}$ reported an increase of 2-5 times in abrasive sliding wear resistance of quenched and tempered steel when the grain size of steel was reduced from 100 to $10 \mu \mathrm{m}$. However, there is little work reported in the literature on the influence of hot rolling and tempering on the particle erosion of martensitic stainless steels. Thus, this study examines the effect of hot rolling and subsequent tempering on the erosion behavior of SUS403 MSS. Also investigated is the correlation between erosion rate and grain size as well as mechanical properties.

\section{Experimental Details}

\subsection{Experimental material and mechanical properties}

The experimental material with similar hot rolling and subsequent tempering has been studied in a previous study. ${ }^{18)}$ After rolling and tempering at $773 \mathrm{~K}$, the material results in higher mechanical properties and in a good balance between hardness and ductility. Thus, the tempering temperature chosen for this study was $773 \mathrm{~K}$. Only brief descriptions are given here to outline its treating process and mechanical properties. The chemical composition of SUS403 MSS is Fe-0.1C-13.17Cr-0.39Si-0.48Mn-0.02P-0.003S. Slabs with three thickness $(16.5,20$ and $28 \mathrm{~mm})$, were prepared to roll for three reductions $(15,30$ and $50 \%)$ in a final thickness of $14 \mathrm{~mm}$, respectively. These slabs were first homogenized to eliminate segregation by holding at $1273 \mathrm{~K}$ for $24 \mathrm{~h}$, and then air cooling down to room temperature. The obtained slabs were preheated to $1283 \mathrm{~K}$ for $2 \mathrm{~h}$, and then removed from the furnace and rolled at an entry temperature of $1173 \mathrm{~K}$. After rolling, the slabs were quenched in water. Finally, these slabs were double-tempered at $773 \mathrm{~K}$. After hot rolling and tempering, at least $2 \mathrm{~mm}$ was machined off to remove the 


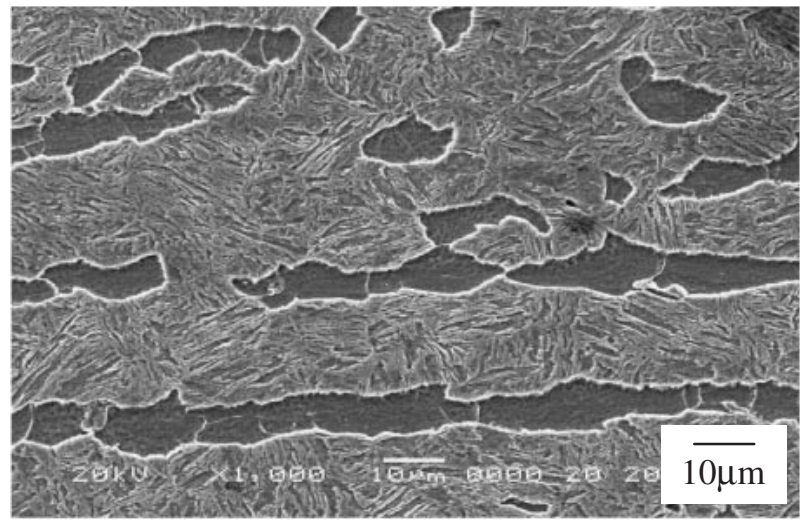

(a)

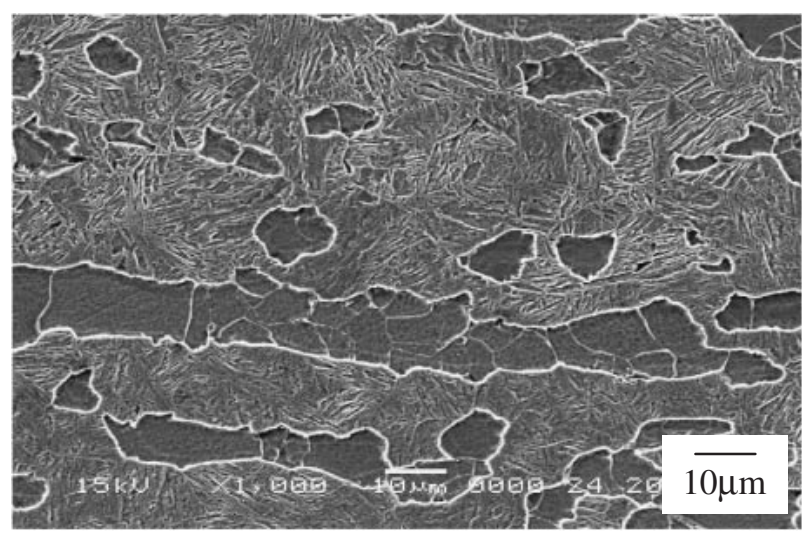

(b)

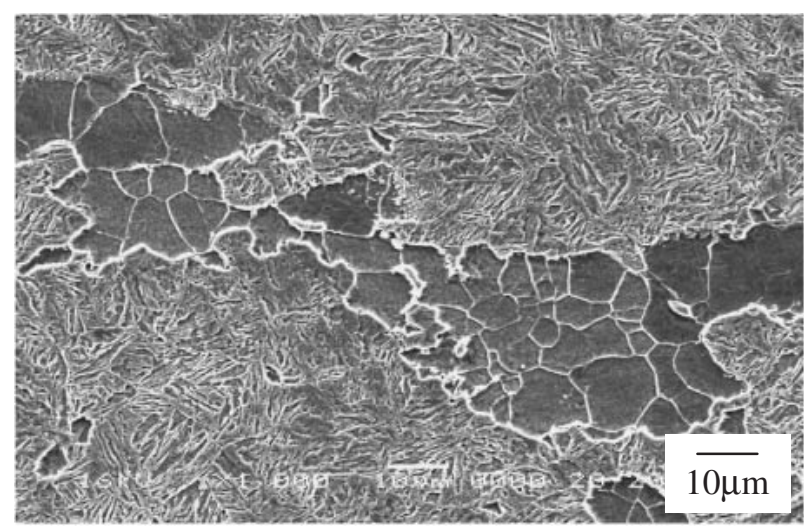

(c)

Fig. 1 SEM metallography of the experimental material after rolling with variant reduction (a) $15 \%$, (b) $30 \%$ and (c) $50 \%{ }^{18)}$

decarburization layer. Then, the slabs were cut into $50 \mathrm{~mm} \times$ $35 \mathrm{~mm} \times 6 \mathrm{~mm}$ shapes parallel to the previous rolling direction for erosion tests. In order to investigate the effect of reduction on erosion rate, one set of slabs was treated as direct quenched from $1283 \mathrm{~K}$ and tempered at $773 \mathrm{~K}$ without rolling for comparison, measuring result of its grain size is $28.6 \mu \mathrm{m}$.

The resulted microstructures of SUS403 MSS rolled with three reductions are shown in Fig. 1. The matrix consists of tempered martensite and ferrite. The lining up of the strainfree and equiaxed ferrite grains is in agreement with the
Table 1 Grain size and mechanical properties of the experimental material after rolling with different reduction and tempering at $773 \mathrm{~K}$.

\begin{tabular}{lrrr}
\hline \multicolumn{1}{c}{ Rolling reduction } & $15 \%$ & $30 \%$ & $50 \%$ \\
\hline Ferrite grain size, $D(\mu \mathrm{m})$ & 19.4 & 13.8 & 8.5 \\
Hardness, $(\mathrm{Hv})$ & 337 & 339 & 368 \\
Ductility, $\varepsilon(\%)$ & 10.3 & 12.6 & 13.9 \\
Tensile strength, $\sigma(\mathrm{MPa})$ & 1150 & 1180 & 1214 \\
Toughness, $E(\mathrm{~J})$ & 29 & 38 & 60 \\
\hline
\end{tabular}

rolling direction. Large reduction results fine grain associated with high density of grain boundary. The results of mechanical properties, including hardness, ductility, ultimate tensile strength and toughness, as well as grain size are shown in Table 1 . It is note that the grain size was only measured on ferrite phase.

\subsection{Erosion test}

The specimens for erosion tests, after the above hot rolling and tempering, were polished with 800 -grit $\mathrm{SiC}$ paper to remove surface scales. These samples were then subjected to erosion wear test. The erosion tests were conducted in a typical sand-blast type of test rig with a $\phi 5 \mathrm{~mm}$ size nozzle. The $\mathrm{Al}_{2} \mathrm{O}_{3}$ particles about $177 \mu \mathrm{m}$ in size and irregular in shape were used as the erodent. Erodent particles were fed to the test rig at a constant rate of $600 \mathrm{~g} / \mathrm{min}$ by using a pressurized carrier gas of $0.3 \mathrm{MPa}$, whereas the erosion velocity at a distance from the nozzle tip of $30 \mathrm{~mm}$ was $83.2 \mathrm{~m} / \mathrm{s}$ estimated by a single-shot high-speed photography. The erosion stream was impinged in the sample surface at different angles of $15,30,45,60$ and $90^{\circ}$; while the amount of erosion particles was $6000 \mathrm{~g}$ for each run. To ensure reproducibility of the result, each erosion test was repeated at least three times. The samples were ultrasonically cleaned in acetone before weighting.

Short-term erosion tests were also conducted on alternative erosion specimens with each impact angle to characterize the erosion wear mode. These samples were pre-polished and pre-etched, then eroded by a total of $5 \mathrm{~g} \mathrm{Al}_{2} \mathrm{O}_{3}$ particles after the compressed air flow reaching steady state. Scanning electron microscopy (SEM) was applied to observe the surface and cross-sectional view of the eroded specimens for the evaluation of the wear mode.

\section{Results and Discussion}

\subsection{Examination of eroded samples}

The worn surfaces of any single certain impact angles for all reduction samples have a similar characteristic, only the $50 \%$ reduction samples were discussed here. The scanning electron micrographs of short-term erosion tests are shown in Fig. 2. The surface of low impact angle $\left(15^{\circ}\right)$ sample shows long and narrow groove, in which cutting chips can be found at the side of the groove, the grooves cut through both the martensite and the ferrite (Fig. 2(a)). The surface of the specimen at the impact angle of $45^{\circ}$ shows a mixture of cutting groove and extrusion crater (Fig. 2(b)). Erosion tests at $60^{\circ}$ and $90^{\circ}$ reveal extrusion of lips and cracks, where the deformation band goes along with the side of lips also 


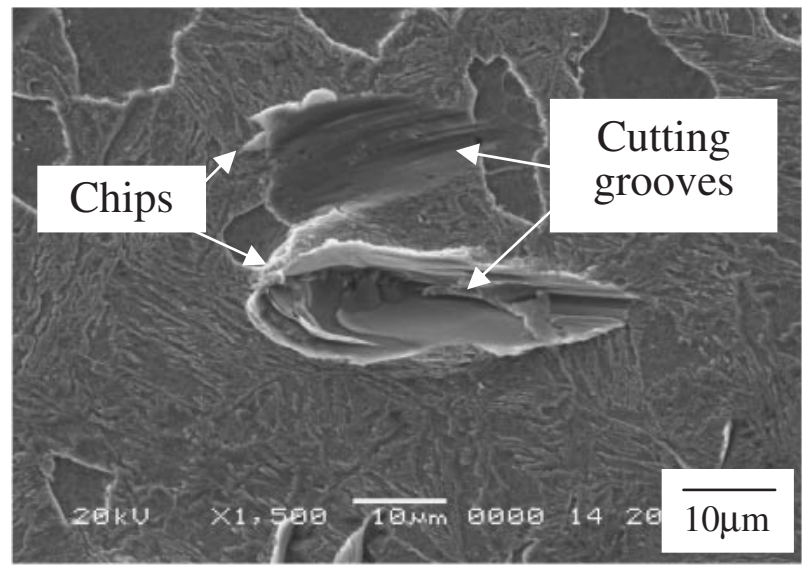

(a)

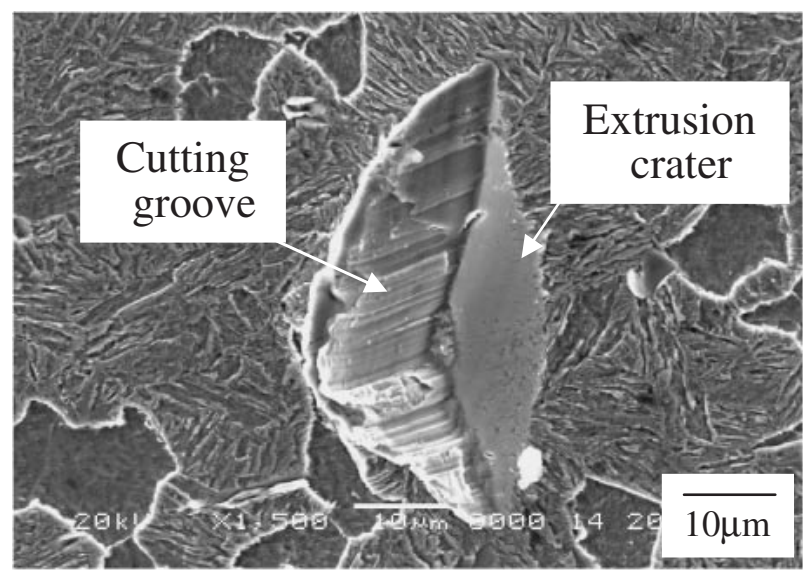

(b)

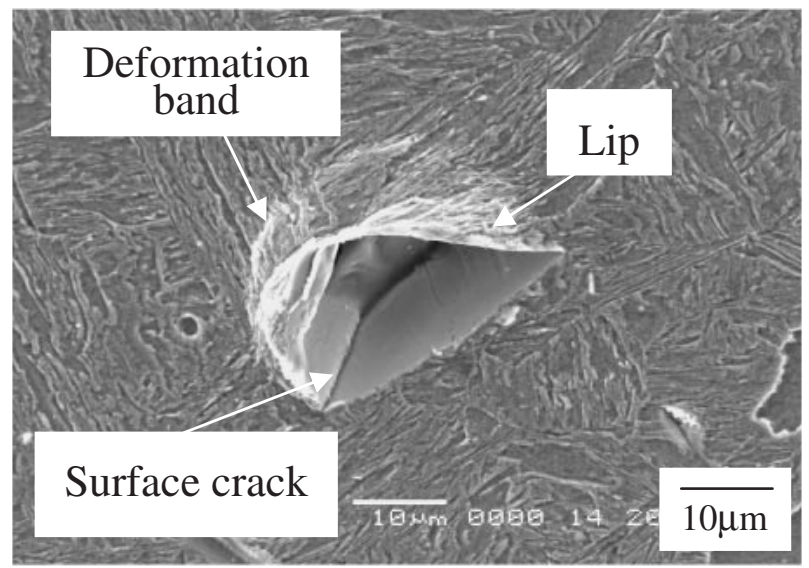

(c)

Fig. 2 SEM micrographs showing the surfaces of samples rolled with $50 \%$ reduction after short-term impingement at variant impact angle (a) $15^{\circ}$, (b) $45^{\circ}$ and (c) $90^{\circ}$.

presented (Fig. 2(c)). It is therefore reasonable to summarize that the wear mode for lower than $30^{\circ}$ is dominated by cutting; for the angle higher than $45^{\circ}$ is based on extrusion and cracking; and a transition of mode mixed by cutting and extrusion occurs between 30 and $45^{\circ}$.

The worn surfaces of the eroded specimens are shown in Fig. 3. The results are similar to the short-term erosion tests,

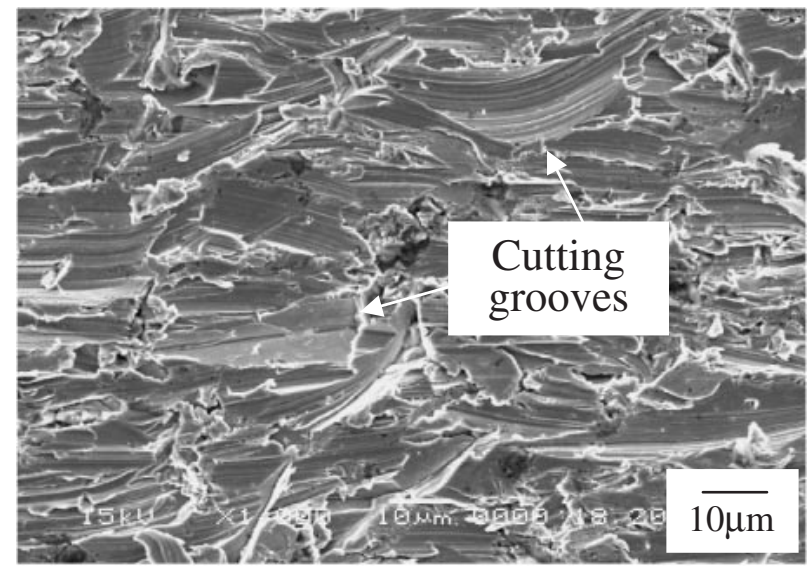

(a)

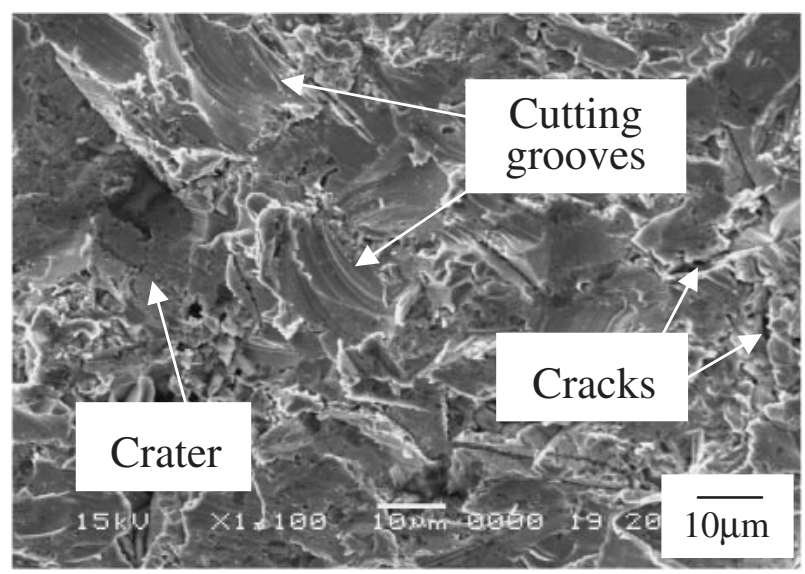

(b)

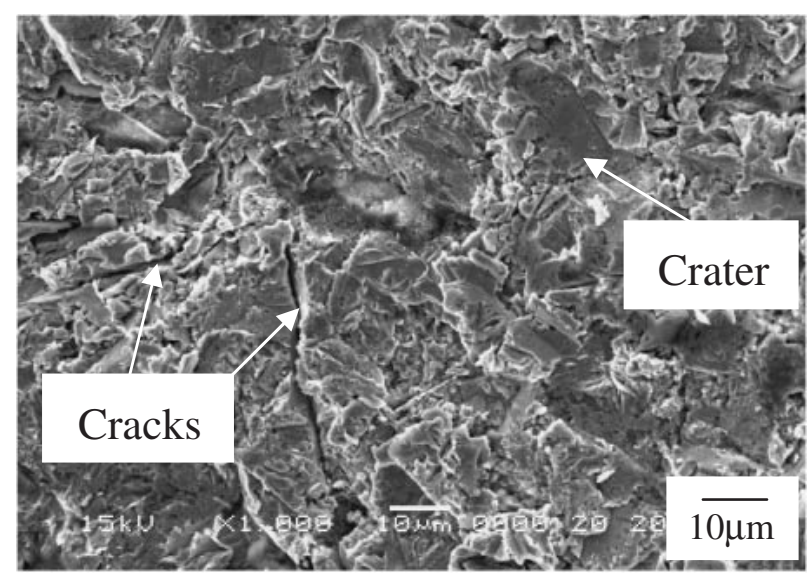

(c)

Fig. 3 SEM micrographs showing the worn surfaces of samples rolled with $50 \%$ reduction after impingement at variant impact angle (a) $15^{\circ}$, (b) $45^{\circ}$ and (c) $90^{\circ}$.

low impact angle erosion causes long and narrow cutting grooves but no surface crack could be observed (Fig. 3(a)). For the medium impact angle, the equal of resolved shear stress and resolved normal stress produces cutting grooves and extrusion lips, where the craters and some surface cracks are found (Fig. 3(b)). In high impact angle, resulting in normal stress, larger scale of surface cracks are observed but 


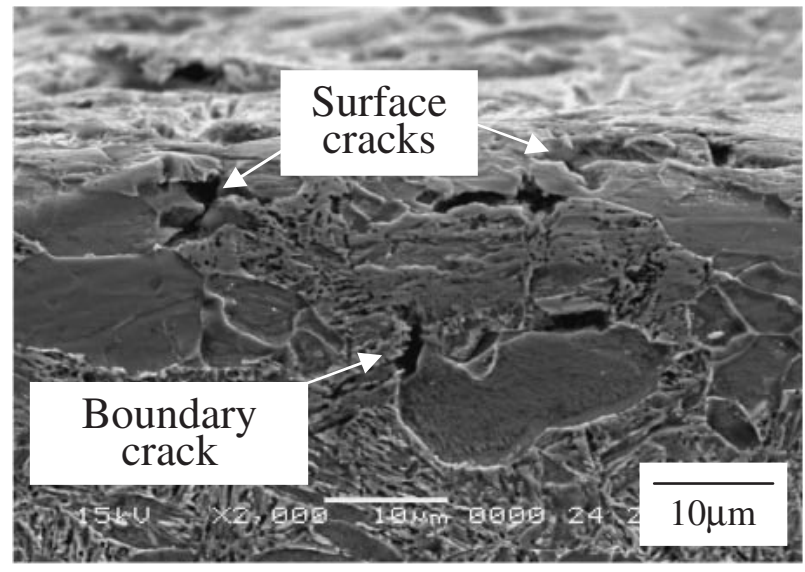

Fig. 4 SEM micrograph showing the cross-sectional view of $50 \%$ reduction sample after impingement at $90^{\circ}$.

cutting mode is absent (Fig. 3(c)). The cross-sectional view of sample impacted at $90^{\circ}$ reveals more information about cracking, as shown in Fig. 4. The metal has undergone severe plastic deformation nearby the surface such that both surface cracks and boundary cracks induced by erodent particle indentation are observed. When these cracks become connected, that causes the material to remove further. After in series observation of overall eroded samples, it clearly shows that the characteristics of cracking are more obvious with increasing impact angle due to the bigger resolved normal stress. Because the grain boundary provides the initiation and propagation sites of cracks, as a result, the erosion rates vary with the grain size. The effects of wear mode, grain size and mechanical properties on erosion rate were further discussed in the following section.

\subsection{Erosion data}

The results of erosion test of samples for all reductions are shown in Fig. 5. The test results of direct quenched and tempered samples also show there for comparison. The variation of erosion rates with impact angle for all reductions exhibit a similar trend, which indicates that the erosion rates first increase with increasing impact angle up to $45^{\circ}$ and then decrease. At lower impact angle $\left(15\right.$ and $\left.30^{\circ}\right)$, the resolved shear stress is higher, that provides a cutting force to take material pieces away. In this case, the cutting groove is shallow and the material loss and erosion rate are lower. For medium impact angle, the resolved shear stress and resolved normal stress is close to equal, those appropriate taking away force to remove the cutting chips and extrusion lips, which in turn raised the erosion rate. For higher impact angle (60 and $90^{\circ}$ ), the resolved normal stress is higher, that provides bigger indented force to forming extrusion lips and cracks but the eroded pieces remove difficult than that impinge at medium and lower angles due to the lower shear stress, and as a consequence, the erosion rates reduce. The maximum erosion rate occurs at the angle of $45^{\circ}$, which shows that the two-phase material mixed with martensite and ferrite causes the angle of maximum erosion rate being neither close to 20 $30^{\circ}$ observed in ductile materials ${ }^{1-4)}$ nor at normal angle reported in brittle materials. ${ }^{5,6)}$

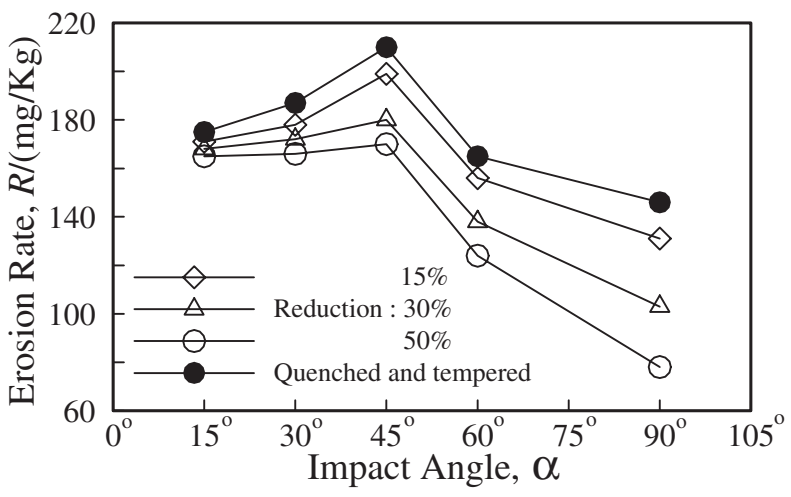

Fig. 5 Erosion rate with respect to impact angle for different reduction samples.

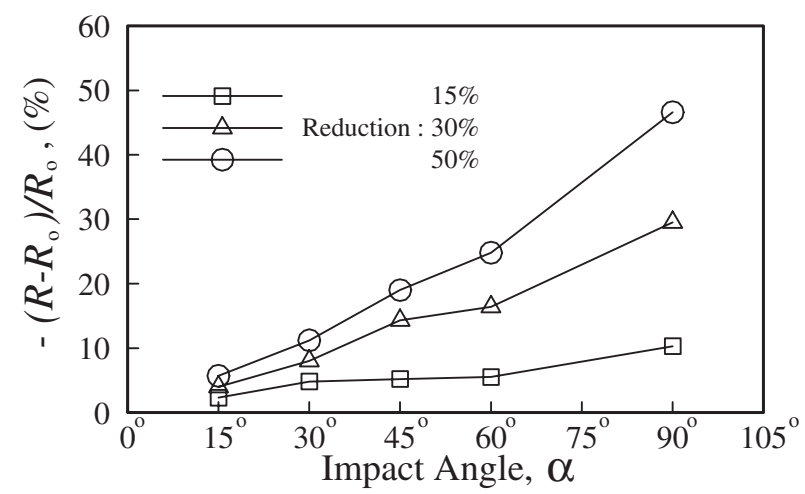

Fig. 6 The percentage of decrement of erosion rate with respect to impact angle in comparison to the direct quenched and tempered samples.

When the effect of reduction is considered, the erosion rates decrease with increasing reduction and are lower than those of direct quenched and tempered samples for all impact angles. These results reveal that the grain refining by hot rolling has an effect on decreasing the erosion rates. The percentage of decrement of erosion rate in comparison to the direct quenched and tempered samples, $-\left(R-R_{o}\right) / R_{o},\left(R_{o}\right.$ : the erosion rate of direct quenched and tempered samples, $R$ : the erosion rate of samples after rolling) is shown in Fig. 6, the percentage of decrement increases with increasing both the impact angle and the reduction. At the normal impact angle, the decrement of erosion rate is approximately $45 \%$ when the material was rolled with $50 \%$ reduction, in which the grain size of the material was reduced from 28.6 to $8.5 \mu \mathrm{m}$. One reason to explain this phenomenon is that the strengthening effect in mechanical properties by grain refining enhances the bonding force of the grain boundaries, thus, the initiation and propagation of the cracks at grain boundary are restricted. Fine grain structure also leads to an increase in the density of grain boundary, which alters the cracking path. Another reason is that the importance of cracking on erosion increases with increasing impact angle. Therefore, taking away the material pieces is decreased with an increase in the reduction and a corresponding increase of the impact angle. On the other hand, coarse grain provides less grain boundaries for impeding crack connection such 


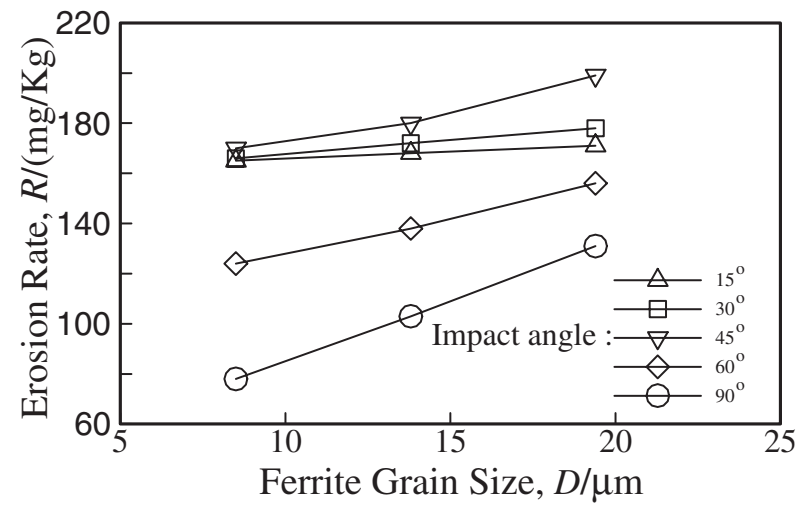

Fig. 7 Erosion rate as a function of ferrite grain size.

that the erosion rate is raised. At lower impact angle (15 and $30^{\circ}$ ), the effects of reduction on decreasing erosion rates decrease because the dominant wear mode is cutting, i.e. the effect of grain refining on erosion rate is only slightly when the cracking is absent. This result suggests that the effects of reduction through their grain refining on decreasing erosion rate are more obvious with an increase of the impact angle.

\subsection{Correlation between erosion rate, grain size and mechanical properties}

The relationship between the erosion rate of the experimental material and its grain size is shown in Fig. 7. At 15 and $30^{\circ}$ of impingement, the grain size appears to have only marginally effect on the erosion rates, because the matrix underwent cutting rather than developed cracks. As described earlier, if cracking is absent, varying the grain size would have slightly effect on the erosion rate, and thus the slop of the curves is lower than the others. However, for impact angle higher than $45^{\circ}$, grain refining significantly decreased the erosion rates. Metallographic evidences reveled that the reason for this erosion behavior is cracks developed under the erosion stream. Once cracks are initiated, these cracks might propagate along the grain boundary (Fig. 4). Fine grain associated with high density of grain boundary and with strengthening effect in mechanical properties retards the crack initiation and alters the cracking path. Consequently, decreasing grain size could lead to lower erosion rates. The slop of the curves increases with increasing impact angle, suggesting that the effect of grain refining on decreasing erosion rate is more obvious when the mainly wear mode is cracking.

Figure 8 shows the plot of erosion rate against hardness and ductility. There is a decrease of erosion rate with an increase in both hardness and ductility. This result is opposite to the observation of previous studies, ${ }^{14,19,20)}$ in which the erosion rate increases with increasing hardness due to the loss in ductility. In this study, increase of hardness also decreases the erosion rate of the material because it retains sufficient ductility. This comparison clearly shows that increasing hardness or strength could decrease the erosion rate of the materials if their ductility or toughness is not lost.

The graphs of erosion rate as function of hardness, ductility, tensile strength and toughness (the last two are not shown) demonstrate that the erosion rate of the exper-

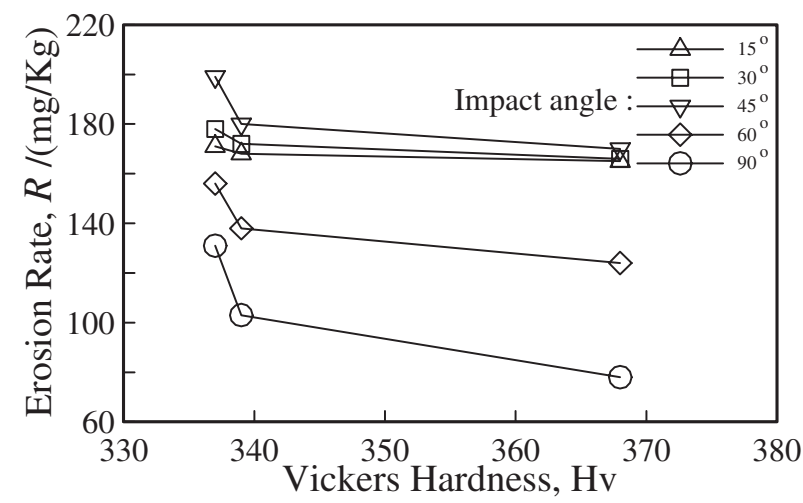

(a)

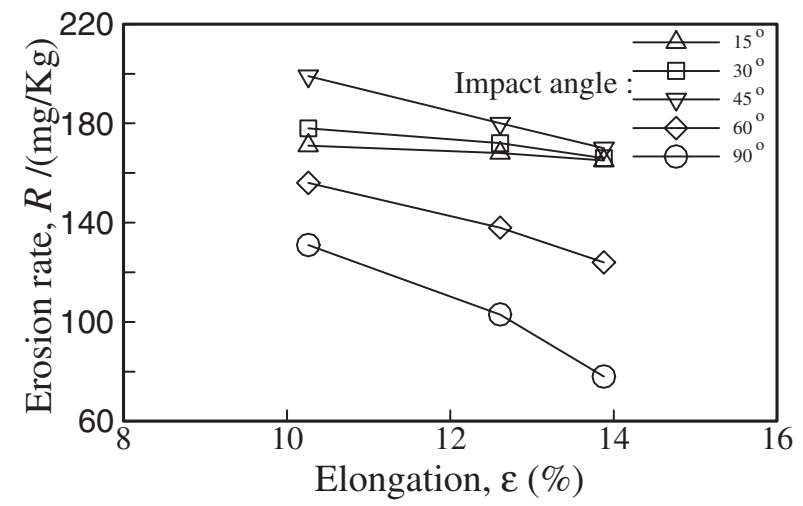

(b)

Fig. 8 Erosion rate as a function of mechanical properties (a) hardness and (b) ductility.

imental material decreases if there is an increase in the mechanical properties due to the decrease of the grain size. All of them are effectively in decreasing the erosion rate of the experimental material.

\section{Conclusions}

The particle erosion behavior of SUS403 MSS after hot rolling and subsequent tempering was investigated as a function of impact angle, reduction and grain size. The erosion rate of the experimental material can be correlated to the grain size and mechanical properties. The results obtained are listed below:

(1) For the experimental material mixed with martensite and ferrite, cutting is the dominant wear mode for material removal at oblique impact angle. At the higher impact angle, the mode is based on extrusion and cracking. However, at the medium impact angle, the wear is dominated by transition of mode mixed with cutting and extrusion. The characteristics of cracking of the worn surface are more obvious with increasing impact angle.

(2) The erosion rates of all reduction samples increase first and then decrease with increasing impact angle. The maximum erosion rate occurs at the angle of $45^{\circ}$ because the equal of resolved shear stress and resolved normal stress appropriate taking away force to remove the material. 
(3) Hot rolling has an effect on decreasing erosion rates due to the grain refining. The percentage of decrement of erosion rate increases with increasing both the impact angle and the reduction. At the normal impact angle, the percentage of decrement of erosion rate is approximately $45 \%$ when the material was rolled with $50 \%$ reduction, in which the grain size was reduced from $28.6 \mu \mathrm{m}$ without rolling condition to $8.5 \mu \mathrm{m}$.

(4) The effect of grain refining on decreasing erosion rate is more obvious when the mainly wear mode is cracking. Consequently, grain refining could decrease the erosion rate at high impact angle, but have slight influence on erosion rate at low impact angle.

(5) For the experimental material, an increase in the mechanical properties including hardness, ductility, tensile strength and toughness due to grain refining are contributed to decreasing the erosion rate.

\section{Acknowledgement}

The author thanks the National Science Council (Taiwan, Republic of China) under Contract NO. NSC 93-2216-E-157001 for financial support.

\section{REFERENCES}

1) I. Finnie: Wear 19 (1972) 81-90.

2) I. M. Hutchings and R. E. Winter: Wear 27 (1974) 121-128.

3) G. Sundararajan: Wear 254 (2002) 129-144.

4) H. Wensink and M. C. Elwenspoek: Wear 254 (2002) 1035-1043.

5) A. W. Ruff and S. M. Wiederhorn: Erosion by solid particle impact, in: C.M. Press (Ed), Treatise on Materials Science and Technology, (Academic Press, New York, 1979), pp. 69-126.

6) J. L. Routbort and R. O. Scattergood: Key Eng. Mater. 71 (1992) 2351.

7) A. Magnee: Wear 181-183 (1995) 500-510.

8) I. Finnie: Wear 186-187 (1995) 1-10.

9) Y. I. Oka, H. Ohnogi, T. Hosokwa and M. Matsumura: Wear 203-204 (1997) 573-579.

10) G. L. Sheldon and I. Finnie: J. Eng. Ind. 88 (1966) 393-400.

11) A. V. Levy: Wear 108 (1986) 1-21.

12) I. M. Hutchings: Wear 35 (1975) 371-374.

13) Y. I. Oka and T. Yoshida: Wear 259 (2005) 102-109.

14) M. Naim and S. Bahadur: Wear 94 (1984) 219-232.

15) K. C. Goretta, R. C. Arroyo, C. T. Wu and J. L. Routbort: Wear 147 (1991) 145-154.

16) G. Bregliozzi, A. Di Schino, S. I. U. Ahmed, J. M. Kenny and H. Haefke: Wear 258 (2005) 503-510.

17) L. G. Korshunov, A. M. Polyakova and N. L. Chrnenko: Umova Vm. Phys. Met. Metallogr. 61 (1986) 160-165.

18) D. C. Wen: Mater. Trans. to be submitted.

19) H. Y. Teng, C. H. Hsu, S. C. Chiu and D. C. Wen: Mater. Trans. 44 (2003) 1480-1487.

20) F. Y. Hung, L. H. Chen and T. S. Lui: Mater. Trans. 42 (2001) 26132621. 